\title{
Energia Metabolizável do Óleo de Soja em Diferentes Níveis de Inclusão para Frangos de Corte nas Fases de Crescimento e Final ${ }^{1}$
}

\author{
Marcelo de Oliveira Andreotti ${ }^{2}$, Otto Mack Junqueira ${ }^{3}$, Maria José Baptista Barbosa ${ }^{4}$, Luciana \\ Cardoso Cancherini ${ }^{5}$, Lúcio Francelino Araújo ${ }^{5}$, Eliana Aparecida Rodrigues ${ }^{5}$
}

RESUMO - Foram conduzidos dois ensaios de metabolismo utilizando-se frangos de corte machos, com o objetivo de determinar o valor de energia metabolizável aparente (EMA) do óleo de soja, o coeficiente de metabolização da matéria seca (CMMS) e a retenção de nitrogênio (RN) em rações isocalóricas, em função de duas idades das aves: crescimento ( 22 - 30 dias) e final (42 - 50 dias) e quatro níveis de inclusão do óleo de soja: 0; 3,3; 6,6 e 9,9\%.Os experimentos tiveram duração de oito dias, sendo três dias para adaptação das aves às rações experimentais e cinco dias para coleta de excretas. $\mathrm{O}$ valor energético do óleo de soja foi determinado por diferença. $\mathrm{O}$ delineamento utilizado nos dois ensaios foi o inteiramente casualizado, com quatro tratamentos e quatro repetições de seis aves cada. Os valores de EMA do óleo de soja, de RN e do CMMS das rações obtidos para os níveis de 3,3; 6,6 e 9,9\% de óleo de soja no período de crescimento foram, respectivamente, de $9.437 \mathrm{kcal} / \mathrm{kg}, 68,22 \mathrm{~g} \mathrm{e} \mathrm{75,47 \% ;} 9307 \mathrm{kcal} / \mathrm{kg}, 74,80 \mathrm{~g} \mathrm{e} 74,69 \%$; e $8.701 \mathrm{kcal} / \mathrm{kg}, 69,98 \mathrm{~g}$ e $74,93 \%$ e para o período final, de $9.558 \mathrm{kcal} / \mathrm{kg}, 54,03 \mathrm{~g}$ e 79,33\%; $8.659 \mathrm{kcal} / \mathrm{kg}, 48,18 \mathrm{~g}$ e $77,92 \%$; e $8307 \mathrm{kcal} / \mathrm{kg}, 52,88 \mathrm{~g} \mathrm{e} 76,28 \%$. Os níveis de inclusão do óleo de soja não influenciaram os valores de EMA, de RN e o do CMMS. A RN foi maior para aves no período de crescimento e o CMMS foi maior para as aves no período final. A EMA do óleo de soja não foi influenciada pelas idades.

Palavras-chave: digestibilidade, frangos de corte, óleo de soja, retenção de nitrogênio, valor energético

\section{Metabolizable Energy of Soybean Oil at Different Inclusion Levels for Broilers in the Growing and Final Phases}

\begin{abstract}
Two experiments of metabolism using broilers were carried out to determine the value for apparent metabolizable energy (AME) of soybean oil, the dry matter metabolization coefficient (DMMC), and the nitrogen retention (NR) in the isocaloric rations on two differents ages: growing phase ( 22 - 30 days old) and final phase (42 - 50 days old) and four inclusion levels of soybean oil: $0 ; 3.3 ; 6.6$ and 9.9\%. The experiments lasted eight days, with three days of adaptation period and five days for excreta collection. The energy values of soybean oil were determined by difference. A completely randomized design, with four treatments and four replications with six birds each, was used. The values of AME of soybean oil, NR and DMMC of the diets obtained at levels of 3.3; 6.6; and 9.9\% of soybean oil in the growing phase were respectively: $9,437 \mathrm{kcal} / \mathrm{kg}, 68.22 \mathrm{~g}, 75.47 \% ; 9,307 \mathrm{kcal} / \mathrm{kg}, 74.80 \mathrm{~g}, 74.69 \%$ and $8701 \mathrm{kcal} /$ $\mathrm{kg}, 69.98 \mathrm{~g}, 74.93 \%$ and to final phase: $9,558 \mathrm{kcal} / \mathrm{kg}, 54.03 \mathrm{~g}, 79.33 \% ; 8,659 \mathrm{kcal} / \mathrm{kg}, 48.18 \mathrm{~g}, 77.92 \%$ and $8,307 \mathrm{kcal} / \mathrm{kg}, 52.88 \mathrm{~g}, 76.28 \%$. The inclusion levels of soybean oil did showed no effect on AME, NR and DMMC values. Higher values of NR and DMMC were obtained, respectively, for the birds in the growing and final phases. Soybean oil AME of was not affected by ages.
\end{abstract}

Key Words: broilers, digestibility, energy value, nitrogen retention, soybean oil

\section{Introdução}

O fator que mais influencia o valor energético dos lipídios é sua digestibilidade, que é dependente do comprimento da cadeia carbônica, do grau de saturação e da posição dos ácidos graxos na molécula de glicerol (Renner \& Hill, 1961; Dvorin et al., 1998).

Além dos fatores físico-químicos relacionados aos lipídios, a correta avaliação de suas verdadeiras contribuições energéticas torna-se extremamente difícil em aves, em função da baixa capacidade fisiológica em digeri-los e utilizá-los, quando ainda muito jovens. Nesse sentido, numerosos estudos têm sido desenvolvidos para investigar a influência da idade das aves na digestão e utilização das diferentes fontes de gordura existentes no mercado.

Carew et al. (1972) demonstraram que a capacidade fisiológica das aves em utilizar gordura

\footnotetext{
${ }^{1}$ Parte da tese de Doutorado do primeiro autor. Bolsista FAPESP. Proc. 00/0256-5.

2 Professor do Departamento de Produção Animal da Universidade Federal de Mato Grosso do Sul - UFMS. E. mail: andreoti@nin.ufms.br

3 Professor do Departamento de Zootecnia da Faculdade de Ciências Agrárias e Veterinárias - FCAV/UNESP.

4 Professora do Departamento de Zootecnia da Universidade Estadual de Maringá - UEM.

${ }^{5}$ Alunos de Pós-graduação da Faculdade de Ciências Agrárias e Veterinárias - FCAV/UNESP.
} 
aumenta com o seu envelhecimento. No entanto, segundo Wiseman \& Salvador (1989), as maiores diferenças na capacidade das aves em utilizar gordura são mais proeminentes entre a primeira e terceira semanas, sendo ausente ou mínima entre a quinta e sétima semanas de vida.

Por outro lado, o nível de inclusão e o tipo de ração utilizados para avaliação das fontes de gordura podem promover diferentes comportamentos quanto à capacidade das aves em utilizá-las, ou seja, dependendo da fonte de gordura e dos níveis de uso na ração, a resposta em termos de sua contribuição energética pode ser linear, curvilínea ou ainda exceder o seu conteúdo em energia bruta (Sibbald \& Kramer, 1978; Wiseman et al., 1986). Isso ficou muito bem demonstrado no estudo conduzido por Dale \& Fuller (1989), que, após realizarem três experimentos para estimar a total contribuição energética dos óleos de milho e de abatedouro avícola, verificaram que os valores de energia metabolizável excediam o conteúdo em energia bruta em ambas as fontes. Os valores obtidos variaram de 8.980 a $10.700 \mathrm{kcal}$ EM/kg para o óleo de milho e de 9.280 a $10.160 \mathrm{kcal}$ EM/kg para o óleo de abatedouro avícola. Resultados semelhantes também foram relatados por Mateos \& Sell (1980a), que, avaliando a graxa amarela e o óleo de soja nas relações de 6:0 a 4:2, encontraram valores entre $8.267 \mathrm{e} 9.717 \mathrm{kcal} \mathrm{EM} / \mathrm{kg}$ para as fontes puras e entre 8.475 e $10.650 \mathrm{kcal} \mathrm{EM} / \mathrm{kg}$ para as misturas. Sibbald \& Kramer (1978) observaram que o valor energético do sebo bovino decrescia linearmente com o aumento de seu nível na ração.

Verifica-se, portanto, que o valor energético das fontes lipídicas é muito variável e pode sofrer influência dos níveis de uso, como também da idade das aves. Objetivou-se, com este trabalho, determinar o valor de energia metabolizável aparente (EMA) do óleo de soja, o coeficiente de metabolização da matéria seca (CMMS) e a retenção de nitrogênio (RN) em rações isocalóricas em função de duas idades das aves e de quatro níveis de inclusão do óleo de soja.

\section{Material e Métodos}

Foram conduzidos dois ensaios de metabolismo com frangos de corte machos da linhagem Cobb. O primeiro, no período de crescimento (22 a 30 dias de idade) e o segundo, no período final (42 a 50 dias de idade), utilizando-se as mesmas aves nos dois ensaios em diferentes idades.
Os frangos foram distribuídos em gaiolas de arame galvanizado, adaptadas com bandejas previamente revestidas com plástico para receber as excretas. Cada gaiola foi equipada com um bebedouro tipo calha, localizado na parte lateral, e um comedouro individual de madeira, na parte frontal.

O delineamento experimental utilizado nos ensaios foi o inteiramente casualizado, com quatro tratamentos e quatro repetição de seis aves cada.

Foram formuladas duas rações sem a adição do óleo de soja - uma para cada fase experimental, segundo recomendações de Rostagno et al. (1994). O óleo de soja foi adicionado às rações em níveis de 0,0 ; 3,$3 ; 6,6$ e 9,9\%, em substituição isocalórica ao amido de milho, de forma a manter constante os outros ingredientes da ração (Tabela 1). A composição químico-bromatológica e o valor energético do óleo de soja e dos demais ingredientes foram calculados segundo recomendações de Rostagno et al. (1994).

Os ensaios foram conduzidos durante oito dias - três para adaptação das aves às rações experimentais e cinco para coleta total das excretas. Após o período de adaptação, foi adicionado óxido férrico (2\%) em todas as rações no primeiro e no último dia como marcador do início e do término da coleta de excretas.

As aves receberam água e ração à vontade durante todo o período experimental. A ração foi fornecida quatro vezes ao dia, a fim de evitar desperdício de ração. As amostras de excretas colhidas foram armazenadas em freezer $\left(-10^{\circ} \mathrm{C}\right)$ até o final do período de coleta. Ao final de cada período experimental, foram anotadas as quantidades de ração consumida e de excreta produzida por repetição.

As excretas depois de descongeladas foram reunidas por repetição, homogeneizadas, retirada uma amostra de cada repetição e colocadas em estufa de ventilação forçada por 72 horas a $55^{\circ} \mathrm{C}$ para pré-secagem. Após a retirada da estufa, estas amostras foram expostas ao ar, para que houvesse o equilíbrio com a temperatura e umidade ambiente; em seguida, foram pesadas, processadas em moinho tipo faca com peneira de $1 \mathrm{~mm}$, sendo então encaminhadas ao laboratório, juntamente com amostras das rações experimentais, para as análises de matéria seca e nitrogênio, realizadas segundo metodologia descrita por Silva (1990).

Os valores de energia bruta das excretas e das rações foram determinados por meio da bomba calorimétrica PARR. A energia metabolizável aparente das rações (EMA) foi determinada com base nos

\section{R. Bras. Zootec., v.33, n.5, p.1145-1151, 2004}


resultados laboratoriais de energia bruta e expressa em $\mathrm{kcal} / \mathrm{kg}$ com base na matéria natural. O valor energético do óleo de soja foi definido por diferença, segundo metodologia descrita por Mateos \& Sell (1981a).
O CMMS das rações experimentais foi determinado dividindo-se o teor de MS obtido da diferença entre a quantidade de MS ingerida e a excretada em cada parcela experimental, pela MS

Tabela 1 - Composição percentual das rações experimentais

Table 1 - Percentual composition of the experimental diets

\begin{tabular}{|c|c|c|c|c|c|c|c|c|}
\hline \multirow[b]{3}{*}{$\begin{array}{l}\text { Ingrediente } / \% \text { de óleo } \\
\text { Ingredient/oil \% }\end{array}$} & \multicolumn{8}{|c|}{$\begin{array}{l}\text { Rações experimentais } \\
\text { Experimental diets }\end{array}$} \\
\hline & \multicolumn{3}{|c|}{$\begin{array}{c}\text { Crescimento } \\
\text { Growing }\end{array}$} & \multirow[b]{2}{*}{9,9} & \multicolumn{4}{|c|}{$\begin{array}{l}\text { Final } \\
\text { Final }\end{array}$} \\
\hline & 0,0 & 3,3 & 6,6 & & 0,0 & 3,3 & 6,6 & 9,9 \\
\hline Milho & 36,80 & 36,80 & 36,80 & 36,80 & 44,05 & 44,05 & 44,05 & 44,05 \\
\hline $\begin{array}{l}\text { Corn } \\
\text { Farelo de soja } \\
\text { Soybean meal }\end{array}$ & 29,80 & 29,80 & 29,80 & 29,80 & 20,05 & 20,05 & 20,05 & 20,05 \\
\hline $\begin{array}{l}\text { Glúten de milho }(60 \%) \\
\text { Corn gluten }(60 \%)\end{array}$ & 5,00 & 5,00 & 5,00 & 5,00 & 8,00 & 8,00 & 8,00 & 8,00 \\
\hline $\begin{array}{l}\text { Óleo de soja } \\
\text { Soybean oil }\end{array}$ & 0,00 & 3,30 & 6,60 & 9,90 & 0,00 & 3,30 & 6,60 & 9,90 \\
\hline $\begin{array}{l}\text { Amido de milho } \\
\text { Corn starch }\end{array}$ & 24,00 & 16,00 & 8,00 & 0,00 & 24,00 & 16,00 & 8,00 & 0,00 \\
\hline $\begin{array}{l}\text { Fosfato bicálcico } \\
\text { Dicalcium phosphate }\end{array}$ & 2,25 & 2,25 & 2,25 & 2,25 & 1,85 & 1,85 & 1,85 & 1,85 \\
\hline $\begin{array}{l}\text { Calcário calcítico } \\
\text { Limestone }\end{array}$ & 1,19 & 1,19 & 1,19 & 1,19 & 1,05 & 1,00 & 1,00 & 1,00 \\
\hline $\begin{array}{l}\text { Sal (Salt) } \\
\text { DL-metionina-99\% }\end{array}$ & $\begin{array}{l}0,30 \\
0,16\end{array}$ & $\begin{array}{l}0,30 \\
0,16\end{array}$ & $\begin{array}{l}0,30 \\
0,16\end{array}$ & $\begin{array}{l}0,30 \\
0,16\end{array}$ & $\begin{array}{l}0,30 \\
0,08\end{array}$ & $\begin{array}{l}0,30 \\
0,08\end{array}$ & $\begin{array}{l}0,30 \\
0,08\end{array}$ & $\begin{array}{l}0,30 \\
0,08\end{array}$ \\
\hline DL-methionine - $99 \%$ & & & & & & & & \\
\hline $\begin{array}{l}\text { L-lisina } \\
L-\text { lysine }\end{array}$ & - & - & - & - & 0,12 & 0,12 & 0,12 & 0,12 \\
\hline $\begin{array}{l}\text { Casca de arroz } \\
\text { Rice hulls }\end{array}$ & 0,00 & 4,70 & 9,40 & 14,10 & 0,00 & 4,75 & 9,45 & 14,15 \\
\hline $\begin{array}{l}\text { Sup. vit. e mineral } * / * * \\
\text { Mineral and vit. supplement } * / * *\end{array}$ & 0,50 & 0,50 & 0,50 & 0,50 & 0,50 & 0,50 & 0,50 & 0,50 \\
\hline $\begin{array}{l}\text { Total } \\
\text { Total } \\
\end{array}$ & 100,00 & 100,00 & 100,00 & 100,00 & 100,00 & 100,00 & 100,00 & 100,00 \\
\hline $\begin{array}{l}\text { Valores calculados } \\
\text { Calculated values }\end{array}$ & & & & & & & & \\
\hline $\begin{array}{l}\mathrm{EM}(\mathrm{kcal} / \mathrm{kg}) \\
M E(k c a l / k g)\end{array}$ & 2.998 & 2.998 & 2.998 & 2.998 & 3.131 & 3.131 & 3.131 & 3.131 \\
\hline PB $(\%)$ & 20,00 & 20,00 & 20,00 & 20,00 & 18,00 & 18,00 & 18,00 & 18,00 \\
\hline $\mathrm{Ca}(\%)$ & 1,07 & 1,07 & 1,07 & 1,07 & 0,90 & 0,90 & 0,90 & 0,90 \\
\hline $\begin{array}{l}\text { P. disponível (\%) } \\
\text { Available P (\%) }\end{array}$ & 0,51 & 0,51 & 0,51 & 0,51 & 0,43 & 0,43 & 0,43 & 0,43 \\
\hline $\begin{array}{l}\text { Metionina+cistina (\%) } \\
\text { Methionine }+ \text { cystine (\%) }\end{array}$ & 0,83 & 0,83 & 0,83 & 0,83 & 0,72 & 0,72 & 0,72 & 0,72 \\
\hline $\begin{array}{l}\text { Lisina }(\%) \\
\text { Lysine }(\%)\end{array}$ & 1,00 & 1,00 & 1,00 & 1,00 & 0,85 & 0,85 & 0,85 & 0,85 \\
\hline
\end{tabular}

* Crescimento. Enriquecimento por quilograma de ração (Growing: Provided per kilogram of ration): Vit. A $9000 \mathrm{UI}$; Vit. E 20,0 mg; Vit. $D_{3} 2200$ Ul; Vit. K 0,5 mg; Vit. $B_{1} 2,0$ mg; Vit. $B_{2} 3,6$ mg; Vit. $B_{12} 20,0$ mcg; Ácido pantotênico (Pantothenic acid) $10,0 \mathrm{mg}$; ácido fólico (Folic acid) 0,5 mg; Nitrovin (Growth promoter) 50,0 mg; Niacina (Niacin) 100,0 mg; Cobre (Cu) 75,0 mg; lodo (I) 1,25 mg; Selênio (Se) 0,25 mg; Manganês (Mn) 120,0 mg; Zinco (Zn) 100,0 mg; Ferro (Fe) 50,0 mg; Antioxidante (Antioxidant) 0,5 mg; Monensina (Coccidicid) $110,0 \mathrm{mg}$.

** Final. Enriquecido por quilograma de ração (Final: Provided per kilogram of ration): Vit. A 1500 UI; Vit. E 1,0 mg; Vit. $\mathrm{D}_{3} 2200$ Ul; Vit. K 0,49 mg; Vit. $\mathrm{B}_{1} 2,0 \mathrm{mg}$; Vit $\mathrm{B}_{2} 1,75 \mathrm{mg}$; Vit. $\mathrm{B}_{12} 5,0 \mathrm{mcg}$; Ácido pantotênico (Pantothenic acid) $10,0 \mathrm{mg}$; Ácido fólico (Folic acid) 0,25 mg; Nitrovin (Growth promoter) 15,0 mg; Niacina (Niacin) 100,0 mg; Cobre (Cupper) 75,0 mg; lodo 1,25 mg; Selênio (Se) 0,25 mg; Manganês (Mn) $120 \mathrm{mg}$; Zinco (Zn) 100,0 mg; Ferro (Fe) $50 \mathrm{mg}$; Antioxidante (Antioxidant) 0,625 mg; Monensina (Coccidicid) 125,0 mg. 
ingerida. A RN foi considerada como a diferença entre o teor de nitrogênio ingerido e o excretado em cada parcela experimental.

Os resultados foram submetidos à análise de grupos de experimentos, segundo Pimentel-Gomes \& Garcia (2002), seguindo um modelo em que os fatores ensaios (Idades) e níveis de óleo foram considerados cruzados. Os parâmetros avaliados foram submetidos a análises de variância e de regressão, por meio do programa estatístico SAEG (1997).

\section{Resultados e Discussão}

Os valores de EMA das rações experimentais calculados e determinados encontram-se na Tabela 2 .

A análise de variância não revelou efeito estatístico $(\mathrm{P}>0,05)$ para os valores de EMA das rações experimentais determinados nos períodos de crescimento e final.

No período de crescimento, pode-se observar que o valor de EMA determinado para a ração referência (sem adição de óleo) foi semelhante ao valor calculado. Esse resultado indica a precisão na determinação dos valores energéticos dos alimentos recomendados pela Tabela Brasileira de Composição Química e Exigência Nutricional para Aves e Suínos (Rostagno et al., 1994). Entretanto, apesar de as rações serem isocalóricas, a adição do óleo de soja proporcionou maior valor energético para as rações que a soma das contribuições energéticas dos seus ingredientes. Os primeiros $3,3 \%$ de inclusão aumentaram em $24 \mathrm{kcal} / \mathrm{kg}$, que corresponde a aumento de $7,27 \mathrm{kcal} / \mathrm{kg}$ para cada $1 \%$ de inclusão do óleo de soja, embora o segundo e terceiro níveis de inclusão tenham resultado em aumentos de $39 \mathrm{e}$ $20 \mathrm{kcal} / \mathrm{kg}$, em relação à ração-referência.

No período final, observa-se que o valor energético determinado para a ração-referência (sem adição de óleo) também foi semelhante ao valor calculado. Ao contrário do que ocorreu no período de crescimento, apenas os primeiros 3,3\% de inclusão do óleo de soja aumentaram o valor energético das rações quando comparados à ração-referência. Esse aumento foi de $24 \mathrm{kcal} / \mathrm{kg}$, semelhante ao valor encontrado no período de 22 a 30 dias. Contudo, para o segundo e terceiro níveis de inclusão, o valor energético das rações experimentais foi reduzido. A queda ocorrida foi de -22 e $-61 \mathrm{kcal} / \mathrm{kg}$, respectivamente, para 6,6 e $9,9 \%$ de inclusão do óleo de soja. Os resultados obtidos nos dois períodos sugerem que os menores níveis de inclusão do óleo de soja foram utilizados mais eficientemente pelas aves, demonstrando que o efeito extracalórico proporcionado pelo óleo de soja foi mais efetivo quando incluído às rações em níveis inferiores a $6 \%$. Valor de EMA determinado maior que o calculado também foi relatado por Sibbald \& Kramer (1978) e Mateos \& Sell (1980a,b).

Os níveis de inclusão do óleo de soja utilizados neste experimento não influenciaram os valores de energia metabolizável e também não houve efeito da interação entre ensaios (idades) e níveis de óleo de soja (Tabela 3). A ausência de diferenças estatísticas neste experimento pode estar relacionada aos elevados

Tabela 2 - Valores energéticos calculados e determinados das rações experimentais para frangos de corte, nos períodos de crescimento e final

Table 2 - Calculated and determined energy values of the experimental diets, for broilers in the growing and final phases

\begin{tabular}{|c|c|c|c|c|}
\hline \multirow{3}{*}{$\begin{array}{l}\text { Níveis de óleo (\%) } \\
\text { Oil levels }(\%)\end{array}$} & \multicolumn{4}{|c|}{$\begin{array}{l}\text { EMA das rações }(\mathrm{kcal} / \mathrm{kg}) \\
\text { AME of the diets }(\mathrm{kcal} / \mathrm{kg})\end{array}$} \\
\hline & \multicolumn{2}{|c|}{$\begin{array}{l}\text { Crescimento } \\
\text { Growing }\end{array}$} & \multicolumn{2}{|c|}{$\begin{array}{l}\text { Final } \\
\text { Final }\end{array}$} \\
\hline & $\begin{array}{l}\text { Calculado } \\
\text { Calculated } \\
\end{array}$ & $\begin{array}{c}\text { Determinado } \\
\text { Determined }\end{array}$ & $\begin{array}{l}\text { Calculado } \\
\text { Calculated } \\
\end{array}$ & $\begin{array}{c}\text { Determinado } \\
\text { Determined } \\
\end{array}$ \\
\hline 0,0 & 2998 & $2999 \pm 19$ & 3131 & $3136 \pm 23$ \\
\hline 3,3 & 2998 & $3023 \pm 04$ & 3131 & $3160 \pm 28$ \\
\hline 6,6 & 2998 & $3038 \pm 21$ & 3131 & $3114 \pm 06$ \\
\hline 9,9 & 2998 & $3019 \pm 48$ & 3131 & $3075 \pm 58$ \\
\hline $\mathrm{CV}(\%)$ & 2,01 & 2,27 & & \\
\hline
\end{tabular}

R. Bras. Zootec., v.33, n.5, p.1145-1151, 2004 
erros-padrão obtidos para os valores médios, dentro de cada nível estudado.

A energia metabolizável verdadeira do sebo bovino decresce linearmente com o aumento de sua inclusão na ração (Sibbald \& Kramer, 1978). Neste experimento, cabe ressaltar que, mesmo não ocorrendo diferença estatística para os níveis de inclusão do óleo de soja, à medida que se aumentou sua inclusão, houve queda numérica nos valores de EMA nos períodos de crescimento e final, verificado pelos valores médios de EMA dentro dos níveis de inclusão $(9497 \pm 518 ; 8983 \pm 186$ e $8504 \pm 363 \mathrm{kcal} / \mathrm{kg}$ ). A redução no valor energético foi maior entre os dois primeiros níveis $(514 \mathrm{kcal} / \mathrm{kg}$ ) que entre os dois últimos $(479 \mathrm{kcal} / \mathrm{kg}$ ). Este resultado indica que, provavelmente, existe interação entre o óleo de soja e os demais ingredientes da ração, como relatado por Sibbald \& Kramer (1978). Por outro lado, a diminuição numérica na EMA do óleo de soja observada neste experimento pode ter ocorrido mais pela redução linear no tempo de trânsito das rações experimentais, observada nos dois períodos de avaliação (Andreotti, 2002), do que propriamente em função da interação com os demais ingredientes. Segundo Mateos \& Sell (1981b), a taxa de passagem do alimento pelo sistema digestório pode modificar a população microbiana do intestino, a capacidade de ingestão e o tempo em que os nutrientes são expostos à ação das enzimas digestivas. Embora a maior taxa de passagem possa ter prejudicado também a digestibilidade dos outros ingredientes da ração, como verificado pela redução numérica nos coeficientes de digestibilidade da matéria seca (Tabela 3), o método utilizado neste experimento inviabilizou a detecção deste efeito, atribuindo-se a variação não-significativa no conteúdo energético das rações somente ao óleo de soja.

É interessante ressaltar que, numericamente, os maiores valores de EMA foram obtidos com 3,3\% de inclusão do óleo de soja. Resultados semelhantes também foram encontrados por Mateos \& Sell (1980b), quando determinaram o valor energético da graxa amarela. Sibbald \& Kramer (1978) relataram que os maiores valores de energia metabolizável proporcionados pelos níveis mais baixos de inclusão foram decorrentes da interação existente entre os ácidos graxos das fontes saturadas em estudo com o teor de ácido linoléico das fontes insaturadas presentes na ração, fato que não se aplica a este experimento, uma vez que o óleo estudado e os alimentos utilizados nas rações foram de origem vegetal.

Com relação à idade (Tabela 3), os resultados obtidos demonstram que não há diferença significativa no valor de EMA do óleo de soja determinado nos períodos de crescimento e final. Trabalhos recentes (Gaiotto et al., 2002, 2003) também demonstraram que a digestibilidade da gordura em frangos de corte é menor somente para a fase pré-inicial, estando diretamente relacionada à reduzida capacidade de produção de lipase pancreática e sais biliares pelas aves nesta fase. Resultados semelhantes aos deste trabalho foram obtidos por Renner \& Hill (1960), os quais demonstraram que a utilização do óleo de milho (insaturado) pelos frangos atingia sua máxima taxa

Tabela 3 - Valores de energia metabolizável aparente (EMA) do óleo de soja, da retenção de nitrogênio e do coeficiente de metabolização da matéria seca das rações experimentais, para frangos de corte nos períodos de crescimento e final

Table 3 - Apparent metabolizable energy values (AME) of soybean oil, nitrogen retention and coefficient of metabolizabble of dry matter of the experimental diets, for broilers in the growing and final phases

\begin{tabular}{|c|c|c|c|c|c|c|}
\hline \multirow{4}{*}{$\begin{array}{l}\text { Níveis de óleo (\%) } \\
\text { Oil levels (\%) }\end{array}$} & \multicolumn{6}{|c|}{$\begin{array}{c}\text { Idade (dias) } \\
\text { Age (days) }\end{array}$} \\
\hline & \multirow{2}{*}{$\begin{array}{l}\text { Crescimento } \\
\text { Growing }\end{array}$} & \multirow{2}{*}{$\begin{array}{l}\text { Final } \\
\text { Final }\end{array}$} & \multirow{2}{*}{$\begin{array}{c}\text { Crescimento } \\
\text { Growing }\end{array}$} & \multirow{2}{*}{$\begin{array}{l}\text { Final } \\
\text { Final }\end{array}$} & \multirow{2}{*}{$\begin{array}{c}\text { Crescimento } \\
\text { Growing }\end{array}$} & \multirow{2}{*}{$\begin{array}{l}\text { Final } \\
\text { Final }\end{array}$} \\
\hline & & & & & & \\
\hline & \multicolumn{2}{|c|}{$\begin{array}{l}\text { EMA do óleo de soja }(\mathrm{kcal} / \mathrm{kg}) \\
\text { AME of soybean oil }(\mathrm{kcal} / \mathrm{kg})\end{array}$} & \multicolumn{2}{|c|}{$\begin{array}{l}\text { Balanço de nitrogênio }(\mathrm{g}) \\
\text { Nitrogen retention }(\mathrm{g})\end{array}$} & \multicolumn{2}{|c|}{$\begin{array}{l}\text { Coeficiente de metabolização da MS (\%) } \\
\text { Dry matter metabolization coefficient }(\%)\end{array}$} \\
\hline$\overline{3,3}$ & $9437 \pm 316$ & $9558 \pm 964$ & 68,22 & 54,03 & 75,47 & 79,33 \\
\hline 6,6 & $9307 \pm 325$ & $8659 \pm 92$ & 74,80 & 48,18 & 74,69 & 77,92 \\
\hline 9,9 & $8701 \pm 413$ & $8307 \pm 595$ & 69,98 & 52,88 & 74,93 & 76,28 \\
\hline $\begin{array}{l}\text { Média } \\
\text { Mean }\end{array}$ & $9148 \pm 210$ & $8841 \pm 364$ & $70,08 \mathrm{~A}$ & $51,88 \mathrm{~B}$ & $75,03 \mathrm{~B}$ & $77,85 \mathrm{~A}$ \\
\hline$\overline{\mathrm{CV}(\%)}$ & \multicolumn{2}{|c|}{11,20} & \multicolumn{2}{|c|}{9,46} & \multicolumn{2}{|c|}{2,43} \\
\hline
\end{tabular}

R. Bras. Zootec., v.33, n.5, p.1145-1151, 2004 
de absorção já aos 28 dias de idade, ao contrário do sebo bovino e da banha suína, que apresentam taxas máximas de utilização a partir dos 42 dias de idade. Isto confirma também os achados de Wiseman et al. (1989), em que as maiores diferenças na capacidade dos frangos em utilizar gorduras ocorrem entre a primeira e terceira semanas de vida, sendo ausente ou mínima entre a terceira, quinta e sétima semanas.

$\mathrm{O}$ valor médio de $8.994 \mathrm{kcal} \mathrm{EM} / \mathrm{kg}$ obtido para o óleo de soja foi inferior ao de $9.201 \mathrm{kcal} / \mathrm{kg}$ determinado por Andreotti (2002), utilizando frangos de corte entre 22 e 30 dias de idade, superior ao de $8.331 \mathrm{kcal} / \mathrm{kg}$ observado por Cardoso et al. (2000), para o óleo de soja degomado, utilizando frangos de mesma idade, e próximo aos valores obtidos por Perez et al. (1989), Brown et al. (1993), Rostagno et al. (1994, 2000) e ao encontrado no NRC (1994). Por outro lado, em função dos elevados erros-padrão da média obtidos dentro dos níveis estudados, verificase a importância de se utilizar mais de um nível de inclusão para se determinar o valor energético das gorduras, como relatado por Wiseman et al. (1986).

Observando-se os valores para o balanço de nitrogênio (Tabela 3 ), verifica-se que não houve efeito do nível de adição do óleo de soja sobre a retenção de nitrogênio nos dois períodos de avaliação. Porém, observa-se que, numericamente, as maiores taxas de retenção de nitrogênio foram obtidas quando foram adicionados $6,6 \%$ e 3,3\% do óleo de soja nos períodos de crescimento e final, respectivamente. Resultado semelhante foi encontrado por Biely \& March (1958), os quais observaram que a adição de 5 e $10 \%$ de sebo bovino em rações com 18 a $22 \%$ de proteína bruta também não influenciou a retenção de nitrogênio, no período de 22 a 28 dias de idade.

Com relação à idade, pode-se observar que a retenção de nitrogênio foi maior para o período de crescimento. Este resultado demonstra que os frangos de corte apresentam maior taxa de crescimento na terceira, quarta e quinta semanas de vida, corroborando os achados de Nitsan et al. (1997) de que a utilização de gordura na terceira e sexta semanas de vida proporciona os melhores resultados de desempenho.

Analisando os valores obtidos para o coeficiente de metabolização da matéria seca (Tabela 3), pode-se verificar que não houve efeito do nível de adição do óleo de soja sobre o mesmo nos dois períodos de avaliação; todavia, o coeficiente de metabolização da matéria seca foi maior no período de 42 a 50 dias de idade.

\section{Conclusões}

Não houve efeito da idade e dos níveis de inclusão do óleo de soja sobre o seu valor de EMA. Cabe ressaltar ainda que, em decorrência dos elevados erros-padrão da média observados dentro de cada nível estudado neste experimento, é recomendável que se utilize mais de um nível de inclusão para avaliar o óleo de soja quanto ao seu valor energético.

Os valores médios de energia metabolizável aparente do óleo de soja determinados com frangos de corte nos períodos de crescimento e final foram de 9.148 e $8.841 \mathrm{kcal} / \mathrm{kg}$, respectivamente.

O coeficiente de metabolização da matéria seca e a retenção de nitrogênio das rações experimentais não foram influenciados pelos níveis crescentes do óleo de soja, em ambos os períodos. Por outro lado, o coeficiente de metabolização da matéria seca foi maior para o período final e a retenção de nitrogênio, no período de crescimento.

\section{Literatura Citada}

ANDREOTTI, M.O. Valor nutricional de diferentes fontes lipídicas para frangos de corte. Jaboticabal: Universidade Estadual Paulista, 2002. 74p. Tese (Doutorado em Zootecnia) Universidade Estadual Paulista, 2002.

BIELY, J.; MARCH., B. Fat and nitrogen retention in chicks fed diets containing different levels of fat and protein. Poultry Science, v.37, p.1235-1240, 1958.

BROWN, P.K.; POTTER, L.M.; WATKINS, B.A. Metabolizable energy values of soybean oil and hydrogenated soybean oil for broilers. Poultry Science, v.72, n.5, p.794-797, 1993.

CARDOSO, C.C.; GOMES, P.C.; ROSTAGNO, H.S. et al. Determinação da energia metabolizável de alguns óleos e gorduras para pintos de corte de 21 a 30 dias de idade. In: REUNIÃO ANUAL DA SOCIEDADE BRASILEIRA DE ZOOTECNIA, 37., 2000, Viçosa, MG. Anais... Viçosa, MG: Sociedade Brasileira de Zootecnia, 2000. p.269.

CAREW, L.B.; MACHEMER, R.H.; SHARP, R.W. et al. Fat absorption by the very young chick. Poultry Science, v. 51, p.738-742, 1972.

DALE, N.M.; FULLER, H.L. Estimating the energy contribution of fats to pratical diets using a chick bioassay. Nutrition Reports International, v.39, n.5, p.1045-1052, 1989.

DVORIN, A.; ZOREF, Z.; MOKADY, S. et al. Nutritional aspects of hydrogenated and regular soybean oil added to diets of broiler chickens. Poultry Science, v.77, p.820-825, 1998.

GAIOTTO, J.B.; MENTEN, J.F.; RACANICCI, A.M.C. et al. Determinação da energia metabolizável de gorduras para frangos de corte. CONFERÊNCA APINCO DE CIÊNCIA E TECNOLOGIA AVÍCOLAS, Suplemento 5., 2003, Campinas. Anais... Campinas:FACTA, 2003. p. 53.

GAIOTTO, J.B.; MENTEN, J.F.; RACANICCI, A.M.C. et al. Determinação da energia metabolizável de gorduras na fase inicial de frangos de corte. In. REUNIÃO ANNUAL DA SOCIEDADE BRASILEIRA DE ZOOTECNIA, 39., 2002,

\section{R. Bras. Zootec., v.33, n.5, p.1145-1151, 2004}


Recife. Anais... Recife: Sociedade Brasileira de Zootecnia/ CD+, (2002). CD-ROM. Nutrição de não-ruminantes.

MATEOS, G.G.; SELL, J.L. Influence of carbohydrate and supplemental fat source on the metabolizable energy of the diet. Poultry Science, v.59, p.2129-2135, 1980a.

MATEOS, G.G.; SELL, J.L. Influence of fat and carbohydrate source on rate of food passage of semipurified diets for laying hens. Poultry Science, v.60, p.2114-2119, 1981 b.

MATEOS, G.G.; SELL, J.L. Metabolizable energy of supplemental fat as related to dietary fat level and methods of estimation. Poultry Science, v. 60, p.1509-1515, 1981a.

MATEOS, G.G.; SELL, J.L. True and apparent metabolizable energy value of fat for laying hens: Influence of level use. Poultry Science, v.59, p.369-373, 1980 b.

NATIONAL RESEARCH COUNCIL - NRC. Nutrient requirements of poultry. 9.rev.ed. Washington, D.C.: National Academy Press, 1994. 155p.

NITSAN, Z.; DVORIN, A.; ZOREF, Z. et al. Effect of added soyabean oil and dietary energy on metabolisable and net energy of broiler diets. British Poultry Science, v.38, n.1, p.101-106, 1997.

PEREZ, A.L.M.; DIAZ, A.J.F.; CEJAS, M.M.A. et al. Energetic evalatuation of different feedstuffs for broiler chickens. Archivos de Zootecnia, v.38, p.21-30, 1989.

PIMENTEL-GOMES, F.; GARCIA, C.H. Estatística aplicada a experimentos agronômicos e florestais: exposição com exemplos e orientações para uso de aplicativos. Piracicaba: Fundação de Estudos Agrários Luiz de Queiroz, 2002. 309p.

RENNER, R.; HILL, F.W. Factors affecting the absorbability of saturated fatty acids in the chick. Journal Nutrition, v.74, p.254-258, 1961.

RENNER, R.; HILL, F.W. The utilisation of corn oil, lard and tallow by chickens of various ages. Poultry Science, v.39, p. 849-854, 1960 .
ROSTAGNO, H.S.; SILVA, D.J.; COSTA, P.M.A. et al. Composição de alimentos e exigências nutricionais de aves e suínos (Tabelas brasileiras). Viçosa, MG: Universidade Federal de Viçosa, 1994. 59p.

ROSTAGNO, H.S.; SILVA, D.J.; COSTA, P.M.A. et al. Tabelas brasileiras para aves e suínos: composição de alimentos e exigências nutricionais. Viçosa, MG: Universidade Federal de Viçosa, 2000. 141p.

SIBBALD, I.R.; KRAMER, J.K.G. The effect of the basal diet on the true metabolizable energy value of fat. Poultry Science, v.57, p.685-691, 1978.

SILVA, D.J. Análise de alimentos (Métodos químicos e biológicos). 2.ed. Viçosa, MG: Universidade Federal de Viçosa, 1990. 165p.

UNIVERSIDADE FEDERAL DE VIÇOSA - UFV. SAEG Sistemas para análises estatísticas e genéticas. Versão 7.1. Viçosa, MG, 1997. 150p. (Manual do usuário).

WISEMAN, J.; COLE, J.A.; PERRY, F.G. et al. Apparent metabolizable energy values of fats for broiler chicks. British Poultry Science, v.27, n.3, p.561-576, 1986.

WISEMAN, J.; SALVADOR, F. Influence of age, chemical composition and rate of inclusion on the apparent metabolisable energy of fats fed to broilers chicks. Britsh Poultry Science, v.30, n.3, p.653-662, 1989. 